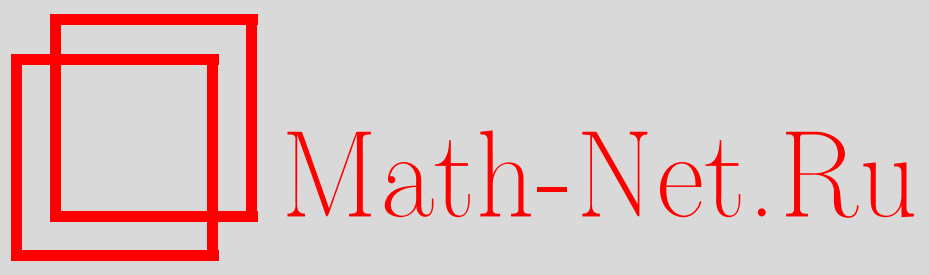

А. В. Павлов, Преобразование Фурье и формула обращения преобразования Лапласа, Матем. заметки, 2011, том 90, выпуск 5, 793-796

DOI: https://doi.org/10.4213/mzm4039

Использование Общероссийского математического портала Math-Net.Ru подразумевает, что вы прочитали и согласны с пользовательским соглашением http://www . mathnet.ru/rus/agreement

Параметры загрузки:

IP: 54.197 .217 .227

26 апреля 2023 г., 11:11:52

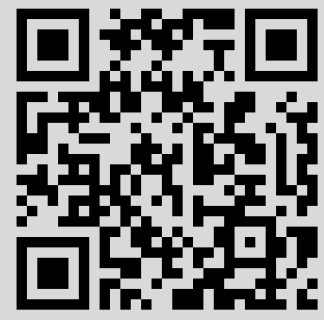




\section{Преобразование Фурье и формула обращения преобразования Лапласа}

\section{А. В. Павлов}

Введение. В данной статье доказано,что мнимая часть аналитического продолжения двойного преобразования Лапласа на отрицательную ось с точностью до константы совпадает с функцией, к которой применено это двойное преобразование Лапласа (следствие 1 к теореме 1) для весьма общего класса функций $Z(x)$ : если

$$
r(s)=\mathscr{L} \mathscr{L}(Z(\cdot))(s), \quad \mathscr{L}(Z(\cdot))(s)=\int_{0}^{\infty} e^{-s x} Z(x) d x, \quad s \in(0, \infty),
$$

то

$$
\pi Z(s)=-\operatorname{Im} r_{\mathrm{An}}(-s), \quad r_{\mathrm{An}}(s) \equiv r(s), \quad s \in(0,+\infty),
$$

где $r_{\mathrm{A} n}(p), p \in \mathbb{C}$, обозначает аналитическое продолжение функции $r(p), p \in D=\{p$ : $\operatorname{Re} p>0\}$, в левую полуплоскость с нарушением аналитичности в, быть может, конечном числе точек. Такое аналитическое продолжение предполагается существующим (аналитическое продолжение через верхнюю полуплоскость) и функция $r_{\mathrm{A} n}(p)$ аналитична на всей действительной оси за исключением, быть может,нуля.

Классу, для которого применим данный результат, как показывает замечание 1 к теореме 1, принадлежат, например, функции вида

$$
\begin{aligned}
Z(x)=\sum_{k=1}^{2 N} \gamma_{k} e^{\lambda_{k} x} & =\sum_{k=1}^{N} \gamma_{k} e^{-\alpha_{k} x} \cos \beta_{k} x, \\
\lambda_{k} & =-\alpha_{k}+\beta_{k} i, \quad \alpha_{k} \in(0, \infty), \quad \gamma_{k}, \beta_{k} \in(-\infty, \infty),
\end{aligned}
$$

при взаимно-сопряженных комплексных корнях

$$
\lambda_{2 j+1}=-\alpha_{2 j+1}+\beta_{2 j+1} i, \quad \lambda_{2 j+2}=-\alpha_{2 j+1}-\beta_{2 j+1} i, \quad j=0, \ldots, N-1 .
$$

Основной результат статьи опирается на теорему 1, в которой приведены условия совпадения

$$
r(s)=-i \mathscr{F}_{0} \mathscr{F}_{0}(Z(\cdot))(s)=\mathscr{L} \mathscr{L}(Z(\cdot))(s), \quad s \in(0,+\infty),
$$

где по определению здесь и далее

$$
\mathscr{F}_{0}(Z(\cdot))(s)=\int_{0}^{\infty} e^{i s x} Z(x) d x, \quad s \in(-\infty, \infty) .
$$

Основной результат. Нам понадобится обозначение $f(p) \in \operatorname{An} G$, если комплексная функция $f(p)$ (вместе со своим аналитическим продолжением) аналитична в открытой области $G \in \mathbb{C}$.

Теорема 1. Если комплексная функция $Z(p)$ такова, что функции

$$
\begin{aligned}
& R(p)=\int_{0}^{\infty} e^{-p x} d x \int_{0}^{\infty} e^{i t x} Z(t) d t \in \operatorname{An} D=\left\{p:-\frac{\pi}{2}<\arg p<\varphi_{0}\right\}, \\
& F(p)=\int_{0}^{\infty} \frac{e^{-x_{1}}}{p} d x_{1} \int_{0}^{\infty} e^{i t x_{1} / p} Z(t) d t \in \operatorname{An} D,
\end{aligned}
$$

при каком-либо $0<\varphi_{0}<\pi / 2$ и непрерывны при всех $p=-i s, s \in(0, \infty)$, то

$$
\mathscr{L} \mathscr{L}(Z(\cdot))(s)=-i \mathscr{F}_{0} \mathscr{F}_{0}(Z(\cdot))(s)=\operatorname{Im} \int_{0}^{\infty} e^{i s x} d x \int_{0}^{\infty} e^{i t x} Z(t) d t, \quad s \in(0, \infty) .
$$

ДокАЗАТЕЛЬСтво. После замены переменной $p x=x_{1}$ в интеграле, определяющем $R(p)$, получим,что $R(p)=F(p), p \in(0, \infty)$. Из совпадения аналитических в открытой обла- 
сти $D$ функций на множестве, имеющем предельную точку внутри этой области (в данном случае это множество совпадает со всей положительной действительной полуосью без нуля),следует совпадение этих функций во всей области аналитичности этих функций: $R(p)=F(p), p \in D$. Так как эти функции непрерывны на границе, то $R(-i s)=F(-i s)$, $s \in(0, \infty)$, и после обратной замены $x_{1} / s=x, s \in(0, \infty), x_{1} \in[0, \infty)$ в интеграле $F(-i s)$ мы получим утверждение теоремы 1.

СледСтвие 1. Пусть функция $\mathscr{F}_{0} \mathscr{F}_{0}(Z(\cdot))(p)=r_{F}(p)$ аналитична в верхней полуплоскости и непрерывна при всех действительных $p \in(-\infty, \infty)$. Если в условиях теоремь функиия $r(s)=\mathscr{L} \mathscr{L}(Z(\cdot))(s), s \in(0, \infty)$, аналитически продолжается в левую полуплоскость через верхнюю полуплоскость с потерей аналитичности в, быть может, конечном числе точек с ненулевой мнимой частъю, то $-\pi Z(x)$ совпадает с мнимой частью аналитического продолжения $r(s)$ на отрицательную действительную полуосъ:

$$
-\pi Z(x)=\operatorname{Im} r_{\mathrm{An}}(-x), \quad x \in(0, \infty), \quad r_{\mathrm{An}}(p)=r(p), \quad p \in D=\{p: \operatorname{Re} p>0\} .
$$

ДокАЗАТЕЛЬСтво. Обозначим

$$
-i r_{F}(p)=-i \mathscr{F}_{0} \mathscr{F}_{0}(Z(\cdot))(p), \quad r_{L}(p)=r_{\mathrm{An}}(p), \quad r_{L}(p)=\mathscr{L} \mathscr{L}(Z(\cdot))(p), \quad p \in(0, \infty) .
$$

Заметим, что действительная часть $r_{F}(s), s \in(-\infty, \infty)$, равна

$$
\begin{aligned}
\operatorname{Re} r_{F}(s) & =\int_{0}^{\infty} \cos s x d x \int_{0}^{\infty} \cos t x Z(t) d t-\int_{0}^{\infty} \sin s x d x \int_{0}^{\infty} \sin t x Z(t) d t \\
& =\frac{1}{4} \int_{-\infty}^{\infty} e^{i s x} d x \int_{-\infty}^{\infty} e^{i t x}\left[Z_{\mathrm{od}}(t)+Z_{\mathrm{ev}}(t)\right] d t \\
& =\frac{1}{4} \int_{-\infty}^{\infty} e^{i s x} d x \int_{-\infty}^{\infty} e^{i t x} Z_{+}(t) d t=\frac{\pi}{2} Z_{+}(-s), \quad s \in(-\infty, \infty),
\end{aligned}
$$

где

$$
\begin{gathered}
Z_{\mathrm{od}}(x) \equiv Z_{\mathrm{ev}}(x) \equiv \frac{Z_{+}(x)}{2}=Z(x), \quad x \in(0,+\infty), \\
Z_{\mathrm{od}}(-x) \equiv Z_{\mathrm{od}}(x), \quad Z_{\mathrm{ev}}(-x) \equiv-Z(x), \quad Z_{+}(-x) \equiv 0, \quad-x \in(-\infty, 0) .
\end{gathered}
$$

Мы проверили, что

$$
\operatorname{Im}\left(-i r_{F}(-s)\right) \equiv-\frac{\pi}{2} Z_{+}(-(-s))=-\pi Z(s), \quad s \in(0, \infty) .
$$

По теореме 1

$$
r_{L}(s)=-i r_{F}(s)=r(s), \quad s \in(0,+\infty) .
$$

Докажем, что эти функции совпадают при всех комплексных $p: \operatorname{Im} p \geqslant 0$.

Обозначим конечное множество точек верхней полуплоскости, в которых нарушается аналитичность какой-либо из функций $r_{L}(p)$ или $r_{F}(p)$, через $Q$. Заметим,что значения функции $\operatorname{ir}_{F}(p)$ действительны при всех положительных, действительных $p$, так как по теореме 1

$$
-i r_{F}(p) \equiv \operatorname{Re}\left(-i r_{F}(p)\right) \equiv \mathscr{L} \mathscr{L}(Z(\cdot))(p)=r(p), \quad p \in(0, \infty) .
$$

Следовательно, по теореме Римана о симметричном продолжении [2; c. 148] функцию

$$
-i r_{F}(p)=-i \mathscr{F}_{0} \mathscr{F}_{0}(Z(\cdot))(p), \quad \operatorname{Im}-i r_{F}(p) \equiv 0, \quad p \in(0, \infty),
$$

действительную при действительных, положительных значения аргумента, можно аналитически продолжить из области $D_{0}=\{p: \operatorname{Im} p>0\}$ в область $D_{1}$, включающую некоторую часть нижней полуплоскости так, что положительная часть действительной полуоси принадлежит некоторой открытой области $D_{1}$ аналитичности этой функции:

$$
(0, \infty) \in D_{1}, \quad-i r_{\mathrm{An}}(p) \in \operatorname{An} D_{1}, \quad D_{0} \in D_{1}, \quad-i r_{\mathrm{An}}(p) \equiv-i r_{F}(p), \quad p \in D_{0} .
$$


Из совпадения $-i r_{\mathrm{An}}(p)=r_{L}(p), p \in(0, \infty) \in D_{1}$ (например, из существования предельной точки совпадения этих функций,принадлежащей открытой области $\left.D_{1}\right)$ следует совпадение $-i r_{\mathrm{An}}(p)=r_{L}(p), p \in D_{1} \backslash Q$. По теореме о симметричном продолжении $-i r_{\mathrm{An}}(p)=-i r_{F}(p)$, $p \in D_{0} \backslash Q \in D_{1}$, так как по предположению функция $-i r_{F}(p) \in \operatorname{An} D_{0} \backslash Q$. Следовательно,

$$
r_{L}(p)=-i r_{\mathrm{An}}(p)=-i r_{F}(p), \quad p \in D_{0} \backslash Q .
$$

По предположению $r_{F}(p)$ определена и непрерывна на действительной оси $p \in(-\infty, \infty)$. Следовательно,

$$
-i r_{F}(-s)=\lim _{p \rightarrow-s}\left(-i r_{F}(p)\right)=\lim _{p \rightarrow-s} r_{L}(p)=r_{L}(-s), \quad s \in(0, \infty) .
$$

Воспользовавшись равенством (0.1), получаем

$$
-\pi Z(s)=\operatorname{Im}\left(-i r_{F}(-s)\right)=\operatorname{Im} r_{L}(-s)=\operatorname{Im} r_{\text {An }}(-s), \quad s \in(0, \infty) .
$$

Следствие 1 доказано.

ЗАмечАние 1. При произвольных целых $l>n+2>1$ (для случая $1<l=n+2$ результаты статьи тоже верны, но проверка аналитичности $\mathscr{F}_{0} \mathscr{F}_{0}(Z(x))$ выходит за рамки данной статьи) выполнено [2; с. 483-485].

$$
\frac{Q_{n}(p)}{P_{l}(p)}=\frac{q_{0}+q_{1} p+\cdots+q_{n} p^{n}}{\prod_{k=1}^{l}\left(p-\lambda_{k}\right)}=2 \pi i \int_{0}^{\infty}\left[\sum_{k=1}^{l} \operatorname{res}_{p=\lambda_{k}} e^{p x} \frac{Q_{n}(p)}{P_{l}(p)}\right] e^{-p x} d x, \quad \operatorname{Re} p \geqslant 0,
$$

если $\lambda_{k} \neq 0, k=1, \ldots, l$.

При любых комплексных $\lambda_{k}=-\alpha_{k}+\beta_{k} i, \alpha_{k} \in(0, \infty), \beta_{k} \in(-\infty, \infty)$ функция

$$
Z(x)=\sum_{k=1}^{l} \gamma_{k} e^{\lambda_{k} x}, \quad \gamma_{k}=\operatorname{res}_{p=\lambda_{k}} \frac{Q_{n}(p)}{P_{l}(p)}, \quad k=1, \ldots, l
$$

удовлетворяет всем условиям теоремы 1 и следствия 1 к данной теореме.

Доказательство. 1. Аналитичность $R(p), p=x+i y$, при всех $x>0, y \in(-\infty, \infty)$, и непрерывность на мнимой оси следует из существования производной от

$$
R(p)=\int_{0}^{\infty} \frac{Q_{n}(t)}{P_{l}(t)} e^{-(x+i y) t} d t
$$

в любой точке $x+i y$ при $x<0$ и, соответственно, равномерной сходимости интеграла $R(x+i y)$ при $x \in[0, \infty), y \in(-\infty, \infty)$.

2. Аналитичность $F(p)$ в области $D=\left\{p=x+i y:-\pi / 2<\arg p<\varphi_{0}\right\}$ при произвольном $0<\varphi_{0}<\min _{0<k<l} \varphi_{k}$, где $\varphi_{k}-$ угол между $\lambda_{k}$ и отрицательной комплексной осью $(0,-i \infty), k=0, \ldots, l$, следует, например, из возможности дифференцировать по $p$ под знаком интеграла в выражении

$$
F(p)=\frac{1}{p} \int_{0}^{\infty} e^{-x_{1}} \frac{Q_{n}\left(-i x_{1} /(x+i y)\right)}{\prod_{k=1}^{l}\left(\left(-i x_{1} /(x+i y)\right)+\alpha_{k}-\beta_{k} i\right)} d x_{1} \in \operatorname{An} D,
$$

так как

$$
\left[\frac{x_{1}}{\sqrt{x^{2}+y^{2}}}\right](-i)(x-i y)=T(-i x-y) \neq-\alpha_{k}+\beta_{k} i
$$

при всех $T \in(0, \infty), p \in D=D_{1} \cup D_{2}$, если по определению $D_{1}=\{x+i y: x>0, y \leqslant 0$, и $D_{2}=\left\{x+i y: x>0, y \geqslant 0,0<\arg (x+i y)<\varphi_{0}\right\}$ (существование подинтегрального выражения при $p \in D_{1}$ следует из положительности $\alpha_{k}>0$ для всех $k$ и неравенства $y \leqslant 0$ 
в $D_{1}$, аналогичное существование подинтегрального выражения при $p \in D_{2}$ следует из совпадения области $D_{2}$ с областью

$$
\left\{-\frac{\pi}{2}-\varphi_{0}<\arg -\frac{i x_{1}}{p}=\arg T(-i x-y)<-\frac{\pi}{2}\right\}
$$

в которой подинтегральная функция аналитична по $p$; равномерная ограниченность производной подынтегрального выражения вытекает из вида функции $e^{-x_{1}} Q_{n}\left(-i x_{1} / p\right) /$ $\left.P_{l}\left(-i x_{1} / p\right)\right)$.

3. Для проверки существования аналитического продолжения $r_{\mathrm{An}}(p)$ через отрицательную действительную полуось изменим порядок интегрирования в выражении $r(p)$ :

$$
r(p)=\mathscr{L} \mathscr{L}(Z(\cdot))(p)=\int_{0}^{\infty} Z(x)\left[\frac{1}{(p+x)}\right] d x \in \mathbb{C} \backslash(-\infty, 0) .
$$

Данная функция аналитична при всех комплексных $p$ кроме отрицательных действительных $p$.

Пусть

$$
p=p_{0} t, \quad t \in(0, \infty), \quad \arg p_{0}=\pi-\varepsilon, \quad 0<\varepsilon \ll 1 .
$$

Методами [1; пп. 2.2-2.3] после замены переменной $x /\left(t p_{0}\right)=z, x \in[0, \infty), t \in(0, \infty)$, получаем

$$
\begin{gathered}
r_{1}\left(p_{0} t\right)=\int_{l\left(p_{0}\right)} Z\left(p_{0} t z\right)\left[\frac{1}{(1+z)}\right] d z \\
t \in(0, \infty), \quad l\left(p_{0}\right)=\left\{z: z=\frac{\tau}{p_{0}}, \tau \in[0, \infty)\right\} \quad\left(l\left(p_{0}\right)=\left\{z: \arg z=\arg \left(\frac{1}{p_{0}}\right)\right\}\right) .
\end{gathered}
$$

Функции

$$
r_{1}(p)=r(p), \quad p=p_{0} t, \quad t \in(0, \infty) ;
$$

функция $r_{1}(p) \in \operatorname{An} D_{\varepsilon}=\left\{p:-2 \varepsilon<\arg p / p_{0}<2 \varepsilon, p \neq 0\right\}$, так как

$$
r_{1}(p)=\int_{l\left(p_{0}\right)} Z(p z)\left[\frac{1}{(1+z)}\right] d z=\int_{0}^{\infty} Z\left(\left(\frac{p}{p_{0}}\right) \tau\right) \frac{1}{1+\left(\tau / p_{0}\right)} d \tau \cdot \frac{1}{p_{0}},
$$

и при $p \in D_{\varepsilon}$ функцию $r_{1}(p)$ можно дифференцировать под знаком интеграла по $p$ ввиду равномерной сходимости интеграла от функции $Z(x)$ из замечания 1 в произвольной малой окрестности $p$, принадлежащей $D_{\varepsilon}$ (в этой окрестности действительная часть $\operatorname{Re}\left(p / p_{0}\right)>$ const $>0)$.

Следовательно, $r_{1}(p)=r(p), p \in l\left(p_{0}\right)$, и функция $r_{1}(p)=r_{A_{n}}(p)$ является аналитическим продолжением функции $r(p)$ через верхнюю полуплоскость в область $D_{\varepsilon},(-\infty, 0) \in D_{\varepsilon}$.

4. Аналитичность $\mathscr{F}_{0} \mathscr{F}_{0}(Z(\cdot))(p)$ в верхней полуплоскости $\{p: \operatorname{Im} p>0\}$ следует из равномерной сходимости интеграла от производной подынтегрального выражения по $p$ в произвольной малой окрестности $p$, принадлежащей этой области, при целых $l>n+2>1$ с учетом $\left|e^{i p t}\right| \leqslant e^{-y t}, p=x+i y, y>0$. Непрерывность данной функции на действительной оси проверяется аналогично.

\section{СПИСОК ЦИТИРОВАННОЙ ЛИТЕРАТУРЫ}

[1] А.В. Павлов, Случайные ряды Фуръе и их применение $к$ теории фильтрации-прогноза, Изд-во Моск. ун-та, М., 2000. [2] М. А. Лаврентьев, Б. В. Шабат, Методъ теории функций комплексного переменного, Наука, М., 1987.

\section{А. В. Павлов}

Поступило

Московский государственный институт радиотехники, электроники и автоматики (технический университет) E-mail: avpavlovmgu@mailfrom.ru

Исправленный вариант 18.05.2011 